\title{
Transmitter Positioning of Distributed Large-Scale Metrology Within Line-Less Mobile Assembly Systems
}

\author{
Christoph Nicksch, Alexander K. Hüttner and Robert H. Schmitt
}

\begin{abstract}
In Line-less Mobile Assembly Systems (LMAS) the mobilization of assembly resources and products enables rapid physical system reconfigurations to increase flexibility and adaptability. The clean-floor approach discards fixed anchor points, so that assembly resources such as mobile robots and automated guided vehicles transporting products can adapt to new product requirements and form new assembly processes without specific layout restrictions. An associated challenge is spatial referencing between mobile resources and product tolerances. Due to the missing fixed points, there is a need for more positioning data to locate and navigate assembly resources. Distributed large-scale metrology systems offer the capability to cover a wide shop floor area and obtain positioning data from several resources simultaneously with uncertainties in the submillimeter range. The positioning of transmitter units of these systems becomes a demanding task taking visibility during dynamic processes and configuration-dependent measurement uncertainty into account. This paper presents a novel approach to optimize the position configuration of distributed large-scale metrology systems by minimizing the measurement uncertainty for dynamic assembly processes. For this purpose, a particle-swarm-optimization algorithm has been implemented. The results show that the algorithm is capable of determining suitable transmitter positions by finding global optima in the assembly station search space verified by applying brute-force method in simulation.
\end{abstract}

\section{Keywords}

Assembly $\cdot$ Large-scale metrology $\cdot$ Transmitter positioning

C. Nicksch ( $\varangle)$ · A.K. Hüttner · R.H. Schmitt

Chair of Production Metrology and Quality Management, RWTH Aachen University, 52074 Aachen, Germany

e-mail: c.nicksch@wzl.rwth-aachen.de

T. Schüppstuhl et al. (eds.), Annals of Scientific Society for Assembly,

Handling and Industrial Robotics 2021,

https://doi.org/10.1007/978-3-030-74032-0_2 


\section{Introduction}

Fluctuating demands as well as increasing number of variants are today's main challenges for manufacturing companies in high-wage countries. To still remain competitive, they face the challenge of producing economically [1]. To transfer market dynamics into long-term competitive advantages, production must be flexible. Especially in the assembly of large-scale products, this is a challenge, since large-scale products are defined by large dimensions at narrow tolerances in the submillimeter range and require disproportionate effort for their manufacturing [2]. One example is the assembly of aircraft, which accounts for more than $50 \%$ of the workload of the entire aircraft manufacturing process [3].

The concept of Line-less Mobile Assembly Systems (LMAS) offers a solution for these challenges and can address new product variants by rapidly reconfiguring assembly stations by integrating mobile resources such as mobilized assembly robots and automated guided vehicles (AGV) for the transport of products [4]. To enable the implementation of LMAS, the mobilization of assembly resources significantly increases the necessary amount of positioning reference data needed on the shop floor. Thus, the need for a metrology infrastructure on the shop floor arises 5]. Distributed large-scale metrology (LSM) consists of multiple transmitter and receiver units and can be adapted to certain assembly processes covering the whole assembly station and providing positioning data with low measurement uncertainties [2]. Transmitter positioning influences the measurement uncertainty significantly and thus limits the maximum measurable tolerance to be checked [6]. As a result, optimal transmitter positions must be determined considering assembly system boundaries and measurement uncertainty.

This paper aims to develop a novel approach to optimize transmitter positions of distributed LSM-systems within LMAS. The approach focuses on an Indoor-GPS-System (iGPS) as an example for distributed LSM-systems. In the following, a brief state-of-the-art for transmitter positioning algorithms and derived algorithm requirements are presented. Subsequently, the new algorithm is described and verification based on a simulated assembly use case is shown.

\section{Distributed Large-Scale Metrology Within Assembly Systems}

\subsection{Approaches for Transmitter Positioning}

Franceschini et al. [7] defines transmitter positioning as a sensor configuration, characterizing each sensor by its spatial coordinates and orientation angles. The problem complexity is strongly related to the geometry of the working environment.

In recent years, there have been multiple publications on the configuration of distributed metrology. Quinders [6] developed a heuristic approach to determine iGPS 
transmitter positions in a 3D space. To reduce complexity, an expert has to manually select potential transmitter positions before an algorithm determines the final configuration in a discretized space. Wang et al. [8] proposed an algorithm for the positioning measurement of laser tracker stations for the error identification of heavy-duty machine tools. He simplifies working ranges using spheres and does not consider dynamics and obstacles (which could collide with the line-of-sight of the laser tracker). Wang and Quinders both use the measurement uncertainty (MU) as the target variable to be minimized. Ray and Mahajan [9] used a genetic algorithm to determine the positions of ultrasonic sensors to track robot positions. Still, he did not consider surrounding obstacles and only focuses on a feasible triangulation during the measurement and not on the MU. The approaches of Galetto and Pralio [10] and Laguna et al. [11] also concentrated on the positioning of ultrasonic sensors. Both approaches considered only a 2D solution space and simplify the solution space by using discretization.

Franceschini et al. [7] summarized that most works have addressed the $3 \mathrm{D}$ sensor positioning problem by considering reduced-size networks, reducing the problem to a $2 \mathrm{D}$ formulation, or referring to simple design goals. Table 1 compares the given approaches. Most of the approaches simplify the solution space and do not take spatial boundaries into account. Still, this is an important factor for metrology systems within LMAS because distributed LSM-systems require a line-of-sight (LOS) between transmitters and receivers. The MU should be considered in particular because it correlates with permitted minimum tolerance of the assembly process.

This work focuses on these deficits and will apply the developed algorithm to a Nikon iGPS measurement system. The iGPS is a suitable metrology system for LMAS scenarios due to its scalability and high accuracy $[2,12]$.

Table 1 Evaluation of approaches for transmitter positioning

\begin{tabular}{|c|c|c|c|c|}
\hline Reference & $\begin{array}{c}\text { Automated } \\
\text { Configuration }\end{array}$ & $\begin{array}{c}\text { Unrestricted } \\
\text { Solution Space }\end{array}$ & $\begin{array}{c}\text { Spatial } \\
\text { Boundaries }\end{array}$ & $\begin{array}{c}\text { Minimizing } \\
\text { MU }\end{array}$ \\
\hline \multicolumn{5}{|l|}{ Quinders } \\
\hline Wang & 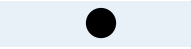 & 0 & 0 & 0 \\
\hline Ray & 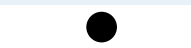 & 0 & 0 & 0 \\
\hline Galetto & & 0 & 0 & 0 \\
\hline Laguna & 0 & $\bigcirc$ & $\bigcirc$ & 0 \\
\hline \multicolumn{5}{|c|}{ O criterion not fulfilled } \\
\hline
\end{tabular}




\subsection{Requirements for iGPS Transmitter Positioning Within LMAS}

The requirements $(\mathrm{R})$ for the iGPS transmitter positioning problem can be categorized as follows:

iGPS: R1: A technically feasible solution depends on the iGPS working range, which is defined by: a permitted distance between a transmitter and a receiver $(2 m-30 m)$, a minimum distance between transmitters $(2 \mathrm{~m})$ and a permitted elevation angle between a transmitter and a receiver $\left( \pm 30^{\circ}\right)$.

R2: To calculate a receiver position, a minimum number of three LOS is required. Figure 1 shows an iGPS configuration with three transmitters and one receiver defined by the elevation angle $\theta$, the azimuth angle $\varphi$ and the transmitter-receiver distance $d$ (corresponds to LOS between transmitters and receivers) [6].

LMAS: R3: The algorithm has to consider all spatial restrictions based on a given LMAS assembly station and its process. This comprises all physical assembly resources involved in the process (e.g. AGVs, robots, products or fixtures) as well as its logical and time-related dependencies.

R4: To enable a simulation close to the real assembly process, complex data formats such as CAD-models need to be considered in terms of resolution and accuracy.

Deficits of the state-of-the-art: R5: Based on the deficits of state-of-the-art approaches, the algorithm should automatically determine a configuration for a distributed LSM system.

R6: The resulting MU is used as an optimization goal and without limiting the solution space and reducing the complexity of the real assembly and measurement environment.

To treat the MU as a target variable of the optimization, a MU-model is required. Quinders [6] developed an empirical MU-model for the iGPS which will be used within this work and explained in detail in Sect. 3.2.

\section{Particle-Swarm-Optimization for Transmitter Positioning}

\subsection{Particle-Swarm-Optimization}

The objective to find a transmitter configuration with the lowest MU for static and dynamic assembly scenarios with several obstacles (assembly resources or products) equals to a restricted and non-steady global optimization problem. For this kind of problems, particle-swarm-optimization has been applied successfully on various benchmark functions and is also used in this work [8, 13, 14].

A transmitter $T$ is defined by its Cartesian coordinates $\mathrm{x}, \mathrm{y}$ and $\mathrm{z}$ (see Eq. 1). The particle swarm consists of $n_{P}$ individual particles where each particle $s$ represents a possible solution. One particle comprises $n_{T}$ transmitters (see Eq. 2). The particle is updated in every iteration $k$ by the term $v$ for $k_{\text {max }}$ iterations (see Eq. 3). The update term $v$ 
in Eq. 4 is based on three scaling parameters $\alpha_{1}, \alpha_{2}$ and $\beta$. Two further variables generate the swarm intelligence: pBest saves the best solution of particle $s$ (cognitive term) and gBest saves the best solution of all particles (social term). The term "best solution" equals the particle with lowest MU. Normally distributed random numbers $U(0,1)$ invoke a stochastic exploration (exploring the whole search space, i.e. assembly stations) and exploitation (converging to local optima). The balance of those properties can be improved by adding a second social parameter $\alpha_{3}$ and a variable lBest which provides information from neighboring particles [13].

$$
\begin{aligned}
& T=\left[\begin{array}{lll}
x & y & z
\end{array}\right] \\
& s_{I}=\left[\begin{array}{lll}
T_{1} & \ldots & T_{n_{T}}
\end{array}\right] \text { with } i \in\left[1, n_{P}\right] \\
& s_{i}^{k+1}=s_{i}^{k}+v_{i}^{k+1} \text { with } k \in\left[1, k_{\max }\right] \\
& v_{i}^{k+1}=\beta \cdot v_{i}^{K}+\alpha_{1} \cdot U(0,1) \cdot\left(p \text { Best }_{i}^{k}-s_{i}^{k}\right)+\alpha_{2} \cdot U(0,1) \\
& \cdot\left(g \text { Best }^{k}-s_{i}^{k}\right)+\alpha_{3} \cdot U(0,1) \cdot\left(\text { lBest }_{i}^{k}-s_{i}^{k}\right)
\end{aligned}
$$

\subsection{Fitness Function and Penalty Terms}

To determine these best solutions, a fitness function $F$ evaluates the particles at each iteration and for all time steps considering dynamic use cases. The fitness function in Eq. 5 contains a target function $f$ and $m$ penalty terms to consider technical constraints (see R1 and R3 in Sect. 2.2). A penalty term comprises a penalty parameter $r$ and a measure of violation $\Psi$ of the constraint. $r$ is set to the amount of a theoretical loss of one LOS at the current solution $s$ since a violation of technical constraints (e.g. measurements out of the working range of the iGPS or within an object) leads to a LOS collision in the worst case.

$$
F\left(s^{k}\right)=f\left(s^{k}\right)+\sum_{i=1}^{m} r\left(s^{k}\right) \cdot \Psi_{i}^{k}\left(s^{k}\right)
$$

If one transmitter is positioned in or above an assembly resource or handling space (prohibited space) at a certain time step, it is defined as a collision of the LOS for the corresponding transmitter-receiver pair. If collisions occur, $\Psi$ is calculated as the number of collisions multiplied by the simulation time steps since a violation at one time step defines the considered configuration unacceptable for the whole process. If a transmitter is positioned too close or too far away from the target, too close to another transmitter or 
exceeds the maximum elevation angle, the Euclidean distance of the exceedances is normalized to the interval from 0 to 1 . The respective $\Psi$ are calculated as the sum of these normalized values over all transmitters. If the minimum number of LOS of the remaining valid transmitter positions is not reached, $\Psi$ is calculated as the difference multiplied by the time steps. If a particle embodies a solution that exceeds the limit of the search space in one dimension, the particle's solution in this dimension is set to its respective limit (decoder approach). If all constraints were satisfied, $\Psi$ for $g B e s t$ would be null.

Quinders [6] determined empirically the MU of the iGPS depending on the positions of the transmitters (see Eq. 6): $\mathrm{u}_{\mathrm{MS}}$ is the resulting MU of the iGPS, $\mathrm{u}_{\mathrm{RE}}$ is the standard uncertainty of the resolution, $\mathrm{u}_{\mathrm{EV}}$ is the standard uncertainty of the equipment variation, $\mathrm{u}_{\mathrm{Ref}}$ is the standard uncertainty of the reference, $\mathrm{u}_{\mathrm{Bi}}$ is the standard uncertainty of the systematic error (bias), $\mathrm{u}_{\mathrm{Cal}}$ is the standard uncertainty of calibration, $\mathrm{u}_{\mathrm{Conf}}$ is the standard uncertainty of the configuration and $\mathrm{u}_{\mathrm{T}}$ is the standard uncertainty of the temperature.

$$
\mathrm{u}_{\mathrm{MS}}^{2}=\max \left\{u_{R E}^{2}, u_{E V}^{2}\right\}+u_{r e f}^{2}+u_{B i}^{2}+u_{\text {Cal }}^{2}+u_{\text {Conf }}^{2}+u_{T}^{2}
$$

Quinders [6] proved that only $\mathrm{u}_{\mathrm{Conf}}$ and $\mathrm{u}_{\mathrm{EV}}$ are affected by transmitter positions. Due to this fact, all remaining constant terms are neglected in Eq. 7 for the optimization problem. According to his experiments, only the average elevation angle $\bar{\theta}(s)$, the maximum azimuth angle $\varphi_{\max }(s)$ and the number of LOS $\mathrm{n}_{\operatorname{los}}(s)$ have impact on the configuration-dependent $\mathrm{MU} f$.

$$
\begin{aligned}
f(s)= & u_{\text {Conf }}^{2}(s)+u_{E V}^{2}(s) \\
= & \left(-11.7 \mathrm{n}_{\operatorname{los}}(s)+87.2\right)^{2}+ \\
& \left(37.9+0.1 \bar{\theta}(s)+0.03 \varphi_{\max }(s)-4,4 \mathrm{n}_{\operatorname{los}}(s)\right)^{2}
\end{aligned}
$$

Based on the solution matrix in Eq. 2 and the fitness function in Eq. 5, the optimization problem for a dynamic assembly with a number of $T_{\max }$ discetized time steps $T_{\mathrm{s}}$ is defined by:

$$
\min _{\mathrm{s}} \frac{1}{T_{\max }} \sum_{j=0}^{\mathrm{T}_{\max }} F_{j}(s)
$$

\subsection{Assembly Simulation for Particle Evaluation and Optimization Procedure}

To calculate the evaluation function $F$, it is necessary to determine $\mathrm{n}_{\mathrm{los}}, \bar{\theta}$ and $\varphi_{\max }$ for all time steps of the assembly process. Therefore, the process is simulated based on CAD-models of the LMAS assembly station. For a first proof-of-concept, assembly resources and products are simplified as convex bodies (boxes or cylinders) to decrease 
the computation time. Positions and rotations of these bodies are updated for each time step using homogeneous transformation matrices. Due to this simplification, a LOS interruption between a receiver-transmitter pair can be detected by calculating an intersection between the connection line and considered physical objects within the assembly station (according to Chazelle and Dobkin [15]).

The integration of the simulation into the optimization problem consists of the following steps: First, the particles are initialized in a random circular distributed manner at middle height in the search space of the assembly station. Afterwards, the fitness function $F$ evaluates all particles for $\mathrm{T}_{\max }$ time steps using the assembly simulation. After determining the terms pBest, gBest and lBest, all particles are updated for the next iteration according to Eq. 3. This cycle repeats until the maximum number of iterations $k_{\max }$ is reached as a stop criterion for the algorithm. After the last iteration, gBest represents the final solution and, accordingly, the transmitter positions with the lowest MU within the particle swarm.

\section{Verification on Assembly Use Case}

\subsection{Description of Simulated Use Case}

The proposed approach is verified by a simulated, theoretical LMAS use case. To match a LMAS scenario, the assembly process contains all relevant types of LMAS resources. The use case is depicted in Fig. 2. The assembly station consists of stationary and mobile components. Three stationary bodies represent fixtures or tooling components. A plane on the floor represents a handling space, reserved for robot movements or logistics, on which it is prohibited to place transmitters. On the left side, an AGV moves along a straight line on the floor. In the center, a robot rotates along two axes simulating an assembly task on a product which is transported by another moving AGV along a circular path on the floor. All mobile resources move simultaneously during the process. An iGPS receiver is placed on the robot tool-center-point (see red dot in Fig. 2). The use case includes a predefined fix set of five transmitters, since more than five LOS do not have a significant impact on the MU [6]. The intention of the use case is to illustrate complex dynamics for a distributed LSM-system within LMAS. The complexity of the system can be further increased by the number and size of additional stationary and mobile resources as well as the simulation time.

\subsection{Analysis of Simulation Results}

To verify if the algorithm is able to detect a global minimum in terms of MU, the solution of the algorithm is compared to a brute-force solution. According to the brute-force method, the $3 \mathrm{D}$ space of the assembly station is discretized using a point-to-point distance 
Fig. 1 iGPS configuration with three transmitters and one receiver according to Quinders [6]

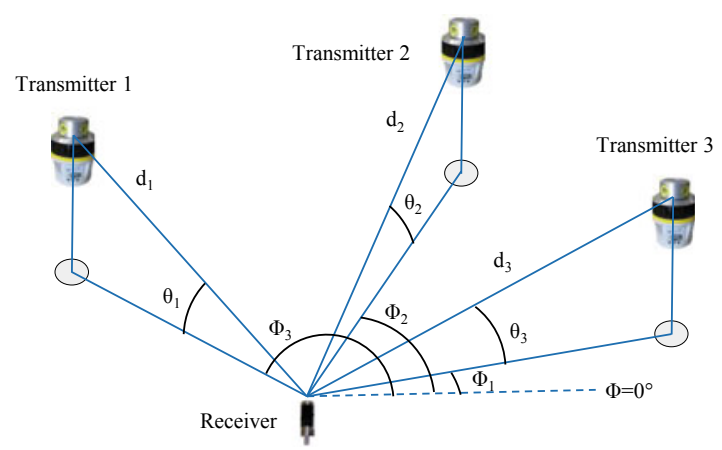

of $2 \mathrm{~m}$. Each point represents a possible position for a transmitter. Based on this solution space, the MU for all possible combination for transmitter configurations are determined by Eq. 7. The transmitter configuration with the lowest MU corresponds to the global optimum as a reference value for the verification of the proposed algorithm.

Table 2 shows the algorithm parameters used within the verification scenario. The parameters $\alpha_{1}, \alpha_{2}, \alpha_{3}$ and $\beta$ decrease by increasing the number of iterations to invoke the exploitation behavior over the process time to find a local minimum through smaller steps (according to Xin et al. [14]). The simulation contains five transmitters using a sample time of $0.1 \mathrm{~s}$. The simulated process takes $7 \mathrm{~s}$.

33 simulation runs were conducted to investigate the performance of the algorithm. Figure 3 shows the best solution after the initialization (red dots), the final best solution after 50 iterations (dark blue dots) out of the 33 simulation runs, the remaining 32 final solutions (light blue dots) and the brute-force solution (yellow dots).

Clearly, the solutions (including the brute-force solution) are arranged in a semicircle around the target trajectory. This can be explained by the fact that the other half is mainly shielded by the robot arm. The three green and two red lines represent the current LOS state $($ green $=$ no collision; red $=$ collision $)$.

Figure 4 shows the resulting MU including the penalty terms over all iterations. After the initialization, the MU of the particle-swarm-optimization solutions lies between $0.3 \mathrm{~mm}$ and $0.5 \mathrm{~mm}$. The amount of penalty terms for each constraint is depicted on the right side. After 16 iterations all constraints referring to the working range of the iGPS are satisfied. After 19 iterations there is no collision between a transmitter and any physical

Table 2 Parameters used within verification

\begin{tabular}{c|cccccc}
$\boldsymbol{n}_{\boldsymbol{P}}$ & $\boldsymbol{k}_{\max }$ & $\boldsymbol{\alpha}_{\mathbf{1}}$ & $\boldsymbol{\alpha}_{\mathbf{2}}$ & $\boldsymbol{\alpha}_{\mathbf{3}}$ & $\boldsymbol{\beta}$ & $\boldsymbol{\Gamma}$ \\
40 & 50 & $2-\frac{1,5}{k_{\max }} * k$ & $1,5-\frac{1}{k_{\max }} * k$ & $2-\frac{1}{k_{\max }} * k$ & $0,9-\frac{0,5}{k_{\max }} * k$ & $\frac{\partial f(s)}{\partial n_{L O S}}$
\end{tabular}



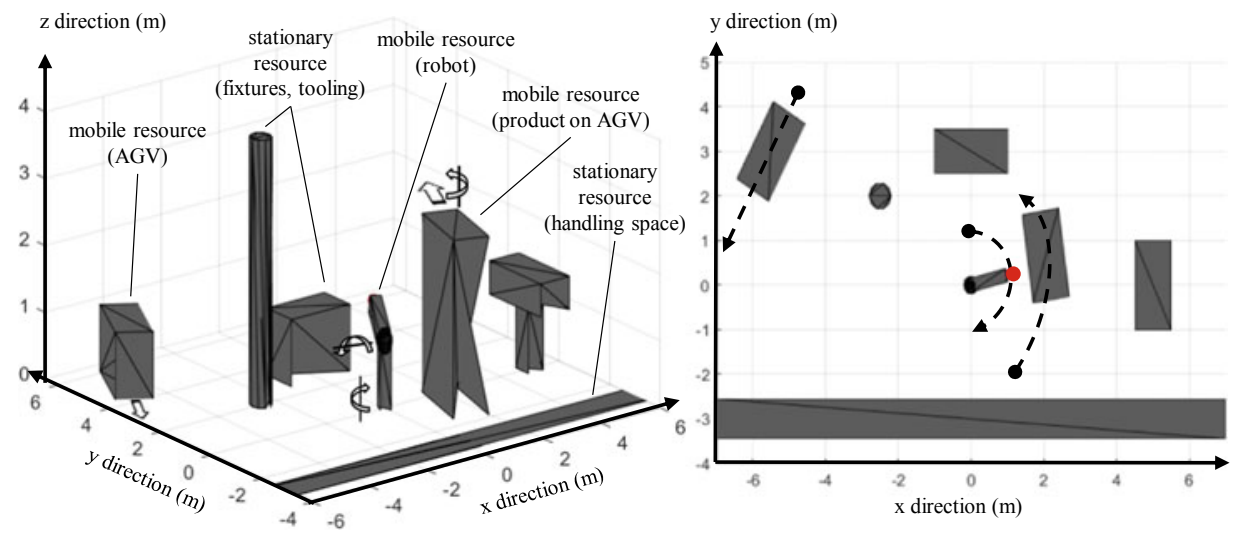

Fig. 2 Isometric view (left) and top view (right) of the LMAS use case

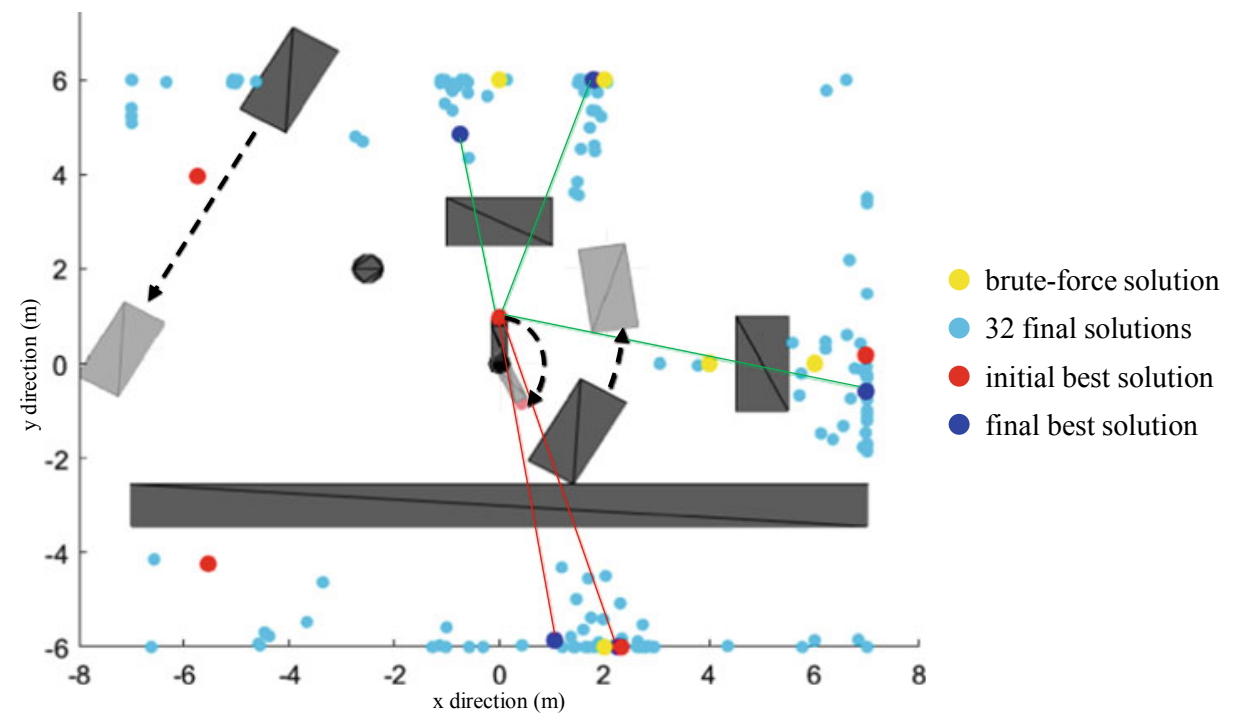

Fig. 3 Visualization of initial, final and brute-force solution with LOS (top view)

object within the assembly station for all 33 simulation runs. The most difficult constraint seems to be the LOS constraint (minimum number of three LOS). After 40 iterations all test runs provide a transmitter configuration with not less than three LOS over the whole process time.

The difference between the brute-force solution $(0.1036 \mathrm{~mm})$ and the median of all 33 particle-swarm-optimization solutions $(0.1044 \mathrm{~mm})$ is $0.0008 \mathrm{~mm}$. The minimum and maximum of the particle-swarm-optimization solutions are $0.1034 \mathrm{~mm}$ and $0.1057 \mathrm{~mm}$ resulting in a span width of $0.0023 \mathrm{~mm}$. It indicates that the algorithm finds even better 

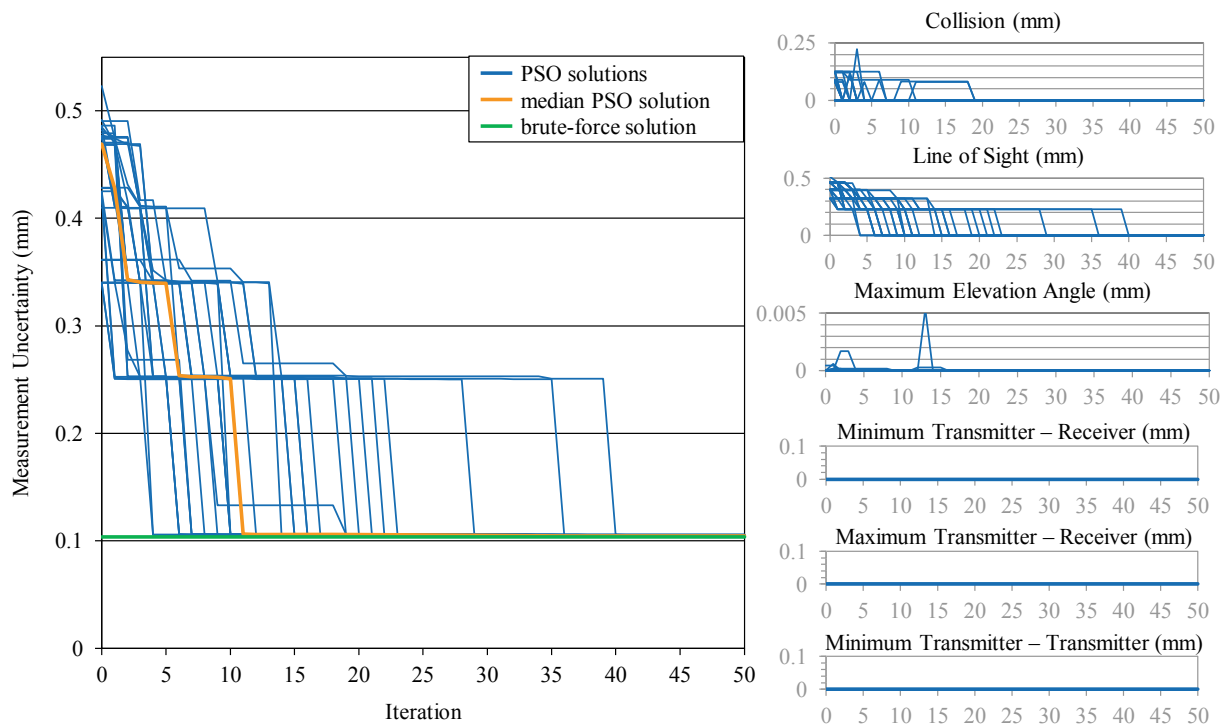

Fig. 4 Development of total costs and penalty terms over 50 Iterations

solutions than the brute-force method. This can be explained by the necessary discretization of $2 \mathrm{~m}$ used for brute-force method.

Referring to the requirements from 2.2, it has been demonstrated that that the proposed algorithm is capable of satisfying all iGPS constraints and thereby fulfils R1 and R2. R3 is satisfied by modeling all relevant LMAS resources (AGVs, robots, products etc.) with their time-dependent positions and orientations during the assembly process. These objects are modeled based on standard CAD-format (e.g. STEP-file) as a composition of convex bodies (R4 is partly fulfilled). The algorithm determines automatically all transmitter positions using the MU as a target variable fulfilling R5 and R6. In contrast to the evaluated research works from 2.1, the solution space (limitation by discretization) and the complexity (2D view) of the possible scenarios is not limited and can be increased as needed and therefore fulfilling R6.

\section{Conclusion and Outlook}

An algorithm for automated transmitter positioning of distributed large-scale metrology within Line-less Mobile Assembly Systems was hitherto unknown to the authors and has been presented in this paper. An iGPS system was used as an example for distributed large-scale metrology. The first implementation of the algorithm, which is based on a particle-swarm-optimization and minimizes the measurement uncertainty shows a good applicability even for a complex and dynamical assembly use case. Simulation results show that the developed algorithm satisfies system constraints (e.g. spatial constraints 
through assembly resources and working ranges of the metrology system). A verification by applying a brute-force method proves that the proposed algorithm converges to the global optimum with a deviation of $0.8 \mu \mathrm{m}$ to the brute-force solution considering a discretized 3D space with a point-to-point distance of $2 \mathrm{~m}$. This approach can also be transferred to other distributed large-scale metrology systems (e.g. laser trackers or laser scanners) by replacing the measurement uncertainty model of the iGPS.

To address the simplification in the simulation by using convex bodies, current research investigates how dynamic octree-collision-detection can be applied to the simulation for line-of-sight analysis in order to enable the import of non-convex bodies with higher resolution. The simulation will be validated through experiments on robot-based assembly stations comparing simulation and experimental results from the real world. Further work includes the enlargement of the iGPS measurement uncertainty model to consider the impact varying distances between transmitters and receivers.

Acknowledgements Funded by the German Federal Ministry for Economic Affairs and Energy (BMWi) within the research project iVeSPA.

\section{References}

1. Beheshti, Z., Shamsuddin, S.M., Sulaiman, S.: Fusion global-local-topology particle swarm optimization for global optimization problems. Mathematical Problems in Engineering (2014)

2. Chazelle, B., Dobkin, D.P.: Intersection of convex objects in two and three dimensions. J. Assoc. Comput. Mach. 34, 1-27 (1987)

3. Franceschini, F., Galetto, M., Maisano, D., Mastrogiacomo, L., Pralio, B.: Distributed Large-Scale Dimensional Metrology. New Insights, p. 85 (2011)

4. Galetto, M., Pralio, B.: Optimal sensor positioning for large scale metrology applications. Precis. Eng. 34(3), 563-577 (2010)

5. Hüttemann, G., Buckhorst, A.F., Schmitt, R.H.: Modelling and assessing line-less mobile assembly systems. Procedia CIRP 81, 724-729 (2019)

6. Laguna, M., Roa, J.O., Jiménez, A.R., Seco, F.: Diversified local search for the optimal layout of beacons in an indoor positioning system. IIE Trans. 41(3), 247-259 (2009)

7. Mei, Z., Maropoulos, P.G.: Review of the application of flexible, measurement-assisted assembly technology in aircraft manufacturing. Proc. Inst. Mech. Eng. Part B: J. Eng. Manuf. 228, 1185 (2014)

8. Montavon, B., Peterek, M., Schmitt, R.: Communication architecture for multiple distributed large volume metrology systems. In: International Symposium on Systems Engineering, pp. 1-2 (2017)

9. Muelaner, J.E., Martin, O.C., Maropoulos, P.G.: Achieving low cost and high quality aero structure assembly through integrated digital metrology systems. Procedia CIRP 7, 688-693 (2013)

10. Muelaner, J.E., Maropoulos, P.G.: Large volume metrology technologies for the light controlled factory. Procedia CIRP 25, 169-176 (2014).

11. Quinders, S.: Virtueller Prototyp zur Optimierung und Absicherung der Konfiguration messtechnisch gestützter und roboterbasierter Montagesysteme. Dissertation, RWTH Aachen University 125-155 (2017) 
12. Ray, P.K., Mahajan, A.: A genetic algorithm-based approach to calculate the optimal configuration of ultrasonic sensors in a 3D position estimation system. Robot. Auton. Syst. 41 (4), 165-177 (2002)

13. Schmitt, R.H., Peterek, M., Morse, E., Knapp W., Galetto, M.: Advances in large-scale metrology - review and future trends. CIRP Ann.-Manuf. Technol. 65(2) 643-665 (2016)

14. Wang, Z., Forbes, A., Maropoulos, P.G. (eds.): Laser tracker position optimization (2014)

15. Xin, J., Chen, G., Hai, Y.: A particle swarm optimizer with multi-stage linearly-decreasing inertia weight. In: International Joint Conference on Computational Sciences and Optimization, p. 505 (2009)

Open Access This chapter is licensed under the terms of the Creative Commons Attribution 4.0 International License (http://creativecommons.org/licenses/by/4.0/), which permits use, sharing, adaptation, distribution and reproduction in any medium or format, as long as you give appropriate credit to the original author(s) and the source, provide a link to the Creative Commons license and indicate if changes were made.

The images or other third party material in this chapter are included in the chapter's Creative Commons license, unless indicated otherwise in a credit line to the material. If material is not included in the chapter's Creative Commons license and your intended use is not permitted by statutory regulation or exceeds the permitted use, you will need to obtain permission directly from the copyright holder.

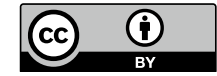

